\title{
Evaluation of the first Coulomb Bridge in three dimensions
}

\author{
Y. Furutani $\left({ }^{*}\right)$, C. Deutsch \\ Laboratoire de Physique des Gaz et des Plasmas (**), Université de Paris-Sud, Centre d'Orsay, 91405 Orsay, France
}

and Y. Oda

Department of Electronics, Faculty of Engineering, Okayama University, Okayama 700, Japan

(Reçu le 14 novembre 1980, révisé le 5 mai 1981, accepté le 7 mai 1981.)

\begin{abstract}
Résumé. - Le premier graphe « Bridge », qui apparaît au 3e ordre du développement nodal, par rapport au paramètre de plasma, de la fonction de corrélations binaires d'équilibre pour le modèle du plasma classique à un composant, est évalué.
\end{abstract}

Abstract. - The first « Bridge » graph, which appears at the third order in the nodal expansion of the equilibrium two-point distribution for the one-component-plasma model, is computed.

The statistical mechanics of the one-componentplasma (OCP) model is presently the subject of much attention because of its relevance to several important dense plasma systems such as those encountered in inertial confinement fusion or in the giant planets (Jupiter, Saturn, ...). The OCP is the simplest classical model of point charges interacting via the Coulomb law in the presence of a neutralizing and mechanically rigid background of opposite sign. It is often used as a testing ground for the various methods recently developed to study equilibrium and transport properties in classical and more realistic strongly coupled Coulomb systems. Of these theoretical techniques, three play a major role :

- The nodal expansion with respect to the classical plasma parameter $\Lambda=e^{2} / k_{\mathrm{B}} T \lambda_{\mathrm{D}}$, in terms of the Debye screening length $\lambda_{\mathrm{D}}$.

- Nonlinear integral equations for the static twopoint pair distribution $g_{2}(x)$.

- Molecular-Dynamics (MD) numerical simulations, of a system containing a few particles.

In practice, these methods work best when used within an interdependent scheme. The resummation up to infinity, based on the skeleton of the convolution chain diagrams with the slowest asymptotic decay, of all the irreducible and short-range graphs

$\left(^{*}\right)$ Permanent address : Department of Electronics, Faculty of Engineering, Okayama University, Okayama 700, Japan.

$\left.{ }^{* *}\right)$ Laboratoire associé au C.N.R.S. into bubble functions yields the so-called hypernetted chain (HNC) nonlinear integral equation for any value, which may be iterated numerically. Thus the corresponding $g_{2}(x)$ data give supplementary information at large $x$, beyond the first two peaks, which cannot be accurately deduced from computer simulations.

In this letter, we address ourselves to an accurate evaluation of the first nonconvolution diagram (Fig. 1), appearing at order $\Lambda^{3}$ in the $\Lambda$-expansion of the potential of average force $w_{2}(x) \equiv \frac{1}{\beta} \cdot \ln g_{2}(x)$.

This is a preliminary, but important, step towards an accurate evaluation of the bubble resummation [1]

$$
\begin{aligned}
& G^{\prime}(K)=G(K)+\sum_{n=3}^{\infty} \Lambda^{3} \beta_{n}^{*}(K)+ \\
& +\sum_{p=2}^{\infty}\left(\frac{K^{2}}{K^{2}+1}\right)^{p-1} \times \sum_{n_{1}, n_{2}, \ldots, n_{p}}^{\infty} \Lambda^{A} \beta_{n_{1}}^{*}(K) \ldots \beta_{n_{p}}^{*}(K),
\end{aligned}
$$

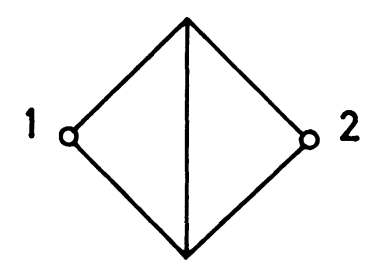

Fig. 1. - Third order Bridge graph. $x$ denotes the relative normalized distance (in units of $\lambda_{\mathrm{D}}$ ) between the root points. 
with $A=\sum_{i=1}^{\infty} n_{i}-1+p$ given in terms of an infinite sum (Iwata-Meeron) of $n$-ladders (parallel) diagrams $G(K)$, and the multiple Bridge quadratures $\beta_{n_{i}}(K)$, in the Fourier transformed space with $K=k \lambda_{\mathrm{D}}$.

Note that all the higher-order $\Lambda^{n}$-Bridge graphs with $n \geqslant 4$ can be constructed from this unique $n=3$ ancestor, by simply joining any pair of topological objects (lines and points) by a Debye line. The HNC resummation of the potential of average force [1] may thus be approximated as

$$
\begin{aligned}
& w_{2}(x)=-\Lambda \frac{\mathrm{e}^{-x}}{x}+\frac{2}{\pi x} \int_{0}^{\infty} \mathrm{d} K K \sin K x \times \\
& \times\left[\frac{K^{2}}{1+K^{2}} \frac{G^{\prime}(K)}{1+\frac{1}{K^{2}}-\frac{1}{\Lambda} G^{\prime}(K)}-G(K)\right] .
\end{aligned}
$$

It should be kept in mind that the present HNC approximation based on an order-by-order resummation is particularly adapted to the $r^{-1}$ interaction. It is at variance with the ad hoc introduction of chain diagrams for arbitrary interaction [8]. This latter does not make full use of the screening effect, which is specific to Coulomb interactions.

In three dimensions, the computation of the Bridge diagrams represents such a formidable task that one is restricted to consider their asymptotic (small $K^{2}$ ) behaviour, which is faster than $\mathrm{e}^{-x} / x$ (Debye) [1], we neglect them by putting $G^{\prime}(K)=G(K)$ in equation (2). This assumption corresponds to the standard HNC approximation. However, an exact evaluation of the Bridge is badly needed to resolve the small but significant discrepancies near the extrema of $g_{2}(x)$ between the HNC results and the Molecular-Dynamics data [3]. It is clear that methods and results similar to those developed here will also apply to higher-order Bridges.

Our goal is achieved by reducing the corresponding 5 -fold quadrature $[4,1]$ first to the double and second to the single integral forms as

$$
\beta_{3}^{*}(x)=-\frac{1}{2 \pi x} \int_{0}^{\infty} \mathrm{d} K K \sin K x I(K),
$$

where

$$
I(K)=\int_{0}^{1} \frac{\mathrm{d} x}{\delta} \int_{0}^{1} \frac{\mathrm{d} y}{\delta^{\prime}} \frac{1}{\left(1+\delta+\delta^{\prime}\right)^{2}+K^{2}(x-y)^{2}},
$$

with

$$
\begin{aligned}
& \delta=\left[1+K^{2} x(1-x)\right]^{1 / 2}, \\
& \delta^{\prime}=\left[1+K^{2} y(1-y)\right]^{1 / 2}
\end{aligned}
$$

This quadrature, first derived by Mitchell and Ninham [4] (see their eq. (A.7)) and examined later by us in the small- $K^{2}$ limit [1], can be transformed into the more tractable form :

$$
\begin{aligned}
& I(K)=\frac{4}{K^{2}} \int_{0}^{\theta_{0}} \frac{\mathrm{d} \varphi}{1+2 \alpha \cos \varphi} \times \\
& \times \arctan \left[\frac{K}{2} \frac{1+2 \alpha \cos \varphi}{1+4 \alpha^{2}+2 \alpha\left(1+\alpha^{2}\right) \cos \varphi}\right],
\end{aligned}
$$

with $\tan \theta_{0}=K / 2$ and $\alpha=\left(1+\frac{K^{2}}{4}\right)^{1 / 2}$. From (4), we readily obtain the small and large $K$ limits as

$$
\begin{aligned}
& I(K) \sim \frac{1}{9}\left(1-\frac{8}{27} K^{2}+\cdots\right), \quad K^{2} \ll 1 \\
& I(K) \equiv I(K)_{\text {asymp. }} \sim \frac{8}{K^{4}}\left(\ln \frac{K}{3}-\frac{1}{3 K}+\cdots\right), \quad K^{2} \gg 1
\end{aligned}
$$

Equation (5a) is identical to our previous result, equation (3.7) of reference [1], while equation $(5 b)$ is new.

To secure a five digit accuracy with $K_{0}=150$, we find it convenient to rewrite equation (3) as

$$
\begin{aligned}
\beta_{3}^{*}(x)= & -\frac{1}{2 \pi x}\left[\int_{0}^{K_{0}} \mathrm{~d} K K \sin K x I(K)\right. \\
& +\int_{K_{0}}^{\infty} \mathrm{d} K K \sin K x\left(I(K)-I(K)_{\text {asymp. }}\right) \\
& \left.+\int_{K_{0}}^{\infty} \mathrm{d} K K \sin K x I(K)_{\text {asymp. }}\right]
\end{aligned}
$$

$\beta_{3}^{*}(x)$ is then computed numerically, using $15000 I(K)$ values in the $0 \leqslant K \leqslant 150$ interval with the aid of the subroutine Fourier at Orsay Univac Centre. Its overall behaviour is given in figure 2 .

A comparison of our results with the available data already obtained by Cohen and Murphy [5], in the range $0.2 \leqslant x \leqslant 3.2$, through an infinite sum involving the Legendre polynomial of the second kind, is displayed in table $\mathrm{I}$.

For $x \gtrsim 3.0$, our calculations confirm

$$
\left|\beta_{3}^{*}(x)\right| \sim \mathrm{e}^{-2 x} / 7 x,
$$

in agreement with the previously conjectured decay $[1,6,7]$ on the basis of topological arguments and the one-dimensional calculations. Near the origin, $\beta_{3}^{*}(x) \sim 0.513(1-3 x)$. Both behaviours support the assumptions displayed above which underly the HNC derivation. Therefore, the first graph $\beta_{3}^{*}(x)$ already exhibits all the expected properties of a Bridge function [1]. The finite value at $x=0$ paves the way to an independent resummation of the $g_{2}(x)$ short-range behaviour through a systematic $n$-ladder resummation. Also we wish to stress the striking analogy 


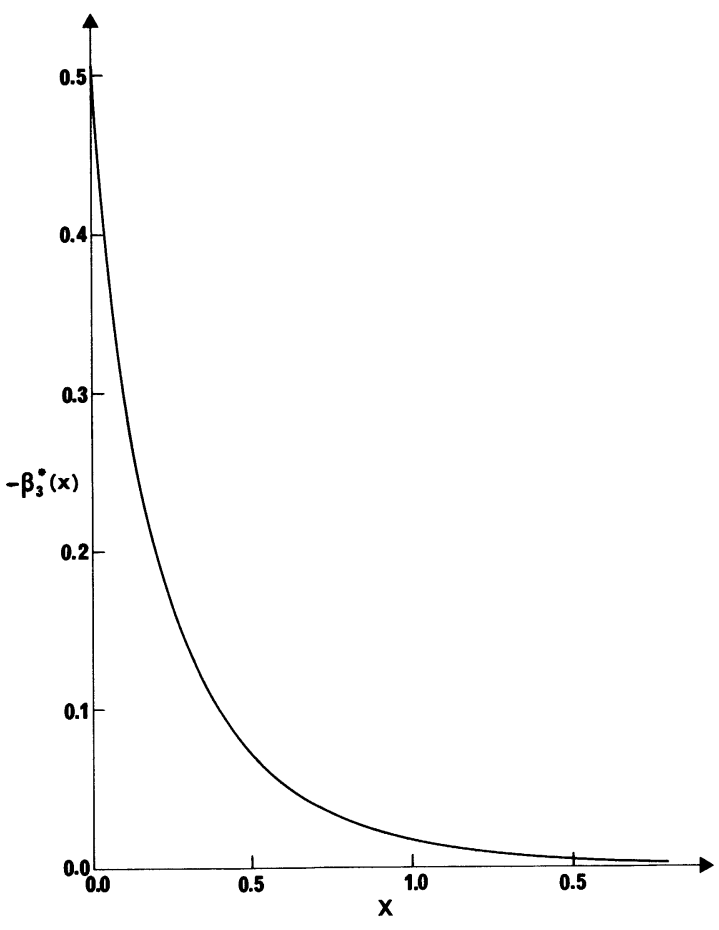

Fig. 2. $-\beta_{3}^{*}(x)$ curve obtained from equations (3) and (4).

between the present three-dimensional $\beta_{3}^{*}(x)$ an its one-dimensional homolog [7]

$\frac{1}{16}\left(1-6 x-\frac{8}{3} \mathrm{e}^{-x}\right) \mathrm{e}^{-2 x} \sim-\frac{3}{8} x \mathrm{e}^{-2 x}, \quad x \geqslant 2$

as far as their asymptotic decay $\sim \mathrm{e}^{-2 x}$, is concerned. To conclude, we note that a numerical computation of equation (1) with $G^{\prime}(K)$, as given explicitly by the present $\beta_{3}^{*}(K)$, is left open for future work.
Table 1. - Results of equation (3) compared with the Cohen-Murphy data [5].

\begin{tabular}{|c|c|c|}
\hline$x$ & $-\beta_{3}^{*}(\mathrm{x})$ & $\begin{array}{c}\text { C.M. Resu1ts } \\
(\text { Ref.5) }\end{array}$ \\
\hline 0.00 & 0.513426 & \\
0.05 & 0.372735 & \\
0.10 & 0.296487 & \\
0.15 & 0.238075 & 0.2002 \\
0.20 & 0.195947 & \\
0.25 & 0.164845 & 0.0984 \\
0.30 & 0.138485 & \\
0.40 & 0.099186 & 0.0529 \\
0.50 & 0.072196 & 0.0300 \\
0.60 & 0.053332 & 0.0168 \\
0.70 & 0.039714 & 0.0098 \\
0.80 & 0.029771 & 0.0058 \\
0.90 & 0.022485 & 0.0034 \\
1.00 & 0.017027 & 0.0021 \\
1.20 & 0.009910 & 0.0013 \\
1.40 & 0.005845 & 0.0007 \\
1.60 & 0.003484 & 0.0005 \\
1.80 & 0.002092 & 0.0003 \\
2.00 & 0.001268 & 0.0002 \\
2.20 & 0.000774 & 0.0001 \\
2.40 & 0.000474 & \\
2.60 & 0.000291 & \\
2.80 & 0.000180 & \\
3.00 & 0.000113 & \\
3.20 & 0.000071 & \\
3.40 & 0.000043 & \\
3.60 & 0.000026 & \\
3.80 & 0.000017 & \\
4.00 & 0.000012 & \\
4.20 & 0.000007 & \\
\hline
\end{tabular}

Acknowledgments. - We thank Dr. Tu Khiet for checking equation (4) and Dr. P. Midy for his advice on numerical computation. The hospitality extended to one of us (YF) by the Laboratoire de Physique des Gaz et des Plasmas, Université de Paris-Sud, Centre d'Orsay, where this work was carried out, is also gratefully acknowledged.

\section{References}

[1] Deutsch, C., Furutani, Y., Gombert, M. M., Phys. Rev. A 13 (1976) 2244 and Phys. Rep. 69 (1981) 85.

[2] Furutani, Y., Deutsch, C., J. Math. Phys. 18 (1977) 292.

[3] Hansen, J. P., Phys. Rev. A 8 (1973) 3096.

[4] Mitchell, D. F., Ninham, B. W., Phys. Rev. 174 (1968) 280.

[5] Cohen, E. G. D., Murphy, T. J., Phys. Fluids 12 (1969) 1404.

[6] Lavaud, M., J. Stat. Phys. 19 (1978) 428.

[7] Deutsch, C., Furutani, Y., Gombert, M. M., J. Phys. A 12 (1979) L 317

[8] Van Leeuwen, J., Groeneveld, J., De Boer, J., Physica 25 (1959) 792. 